\title{
Erratum to: A New Class of Wavelet-Based Metrics for Image Similarity Assessment
}

\author{
Maria Grazia Albanesi $^{1}\left([) \cdot\right.$ Riccardo Amadeo $^{1} \cdot$ Silvia Bertoluzza $^{2}(1) \cdot$ \\ Giulia Maggi ${ }^{3}$
}

Published online: 25 July 2017

(C) Springer Science+Business Media, LLC 2017

\section{Erratum to: J Math Imaging Vis}

\section{DOI 10.1007/s10851-017-0745-1}

The original version of this article unfortunately contained a misprint in Eq. 9. It was published with erroneously repeated vertical bars.

The correct equation is given below:

$\left\|s^{J}\right\|_{2}^{2}=\left\|s^{J-1}\right\|_{2}^{2}+\left\|\mathbf{d}^{J-1}\right\|_{2}^{2}=\left\|s^{0}\right\|_{2}^{2}+\sum_{j=0}^{J-1}\left\|\mathbf{d}^{j}\right\|_{2}^{2}$.

The original article has been corrected.

The online version of the original article can be found under https:// doi.org/10.1007/s10851-017-0745-1.

Silvia Bertoluzza

silvia.bertoluzza@imati.cnr.it

Maria Grazia Albanesi

mariagrazia.albanesi@unipv.it

1 Department of Electrical, Computer and Biomedical Engineering, University of Pavia, Via Ferrata 1, 27100 Pavia, Italy

2 CNR IMATI Enrico Magenes, via Ferrata 1, 27100 Pavia, Italy

3 ICH Humanitas, Milan, Italy 